\title{
Investigating Wilms' Tumours Worldwide: A Report of the OxPLORE Collaboration-A Cross-Sectional Observational Study
}

\author{
K. Ford ${ }^{1}$ - S. Gunawardana ${ }^{2}$ E. Manirambona ${ }^{3}$ - G. S. Philipoh ${ }^{4} \cdot$ B. Mukama ${ }^{3}$ ' \\ A. Kanyamuhunga ${ }^{5}$ P. Cartledge ${ }^{3,6} \cdot$ M. J. Nyoni ${ }^{4} \cdot$ D. Mwaipaya ${ }^{4} \cdot$ J. Mpwaga $^{4}$.

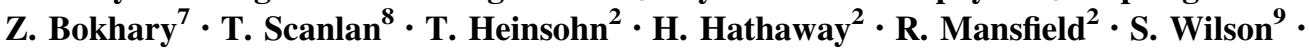 \\ K. Lakhoo ${ }^{1,2,7,9}$
}

Published online: 11 October 2019

(C) The Author(s) 2019

\begin{abstract}
Background Childhood cancer is neglected within global health. Oxford Pediatrics Linking Oncology Research with Electives describes early outcomes following collaboration between low- and high-income paediatric surgery and oncology centres. The aim of this paper is twofold: to describe the development of a medical student-led research collaboration; and to report on the experience of Wilms' tumour (WT).

Methods This cross-sectional observational study is reported as per STROBE guidelines. Collaborating centres included three tertiary hospitals in Tanzania, Rwanda and the UK. Data were submitted by medical students following retrospective patient note review of 2 years using a standardised data collection tool. Primary outcome was survival (point of discharge/death).

Results There were 104 patients with WT reported across all centres over the study period (Tanzania $n=71$, Rwanda $n=26$, UK $n=7$ ). Survival was higher in the high-income institution [ $87 \%$ in Tanzania, 92\% in Rwanda, $100 \%$ in the UK $\left.\left(X^{2} 36.19, p<0.0001\right)\right]$. Given the short-term follow-up and retrospective study design, this likely underestimates the true discrepancy. Age at presentation was comparable at the two African sites but lower in the UK (one-way ANOVA, $F=0.2997, p=0.74)$. Disease was more advanced in Tanzania at presentation $(84 \%$ stage IIIIV cf. $60 \%$ and $57 \%$ in Rwanda and UK, respectively, $X^{2} 7.57, p=0.02$ ). All patients had pre-operative chemotherapy, and a majority had nephrectomy. Post-operative morbidity was higher in lower resourced settings $\left(X^{2}\right.$ $33.72, p<0.0001)$. Methodology involving medical students and junior doctors proved time- and cost-effective. This collaboration was a valuable learning experience for students about global research networks.

Conclusions This study demonstrates novel research methodology involving medical students collaborating across the global south and global north. The comparison of outcomes advocates, on an institutional level, for development in access to services and multidisciplinary treatment of WT.
\end{abstract}

K. Lakhoo

kokila.lakhoo@paediatrics.ox.ac.uk

1 Department of Pediatric Surgery, Oxford University Hospital, Headley Way, Headington, Oxford OX3 9DU, UK

2 Oxford University, Oxford, UK

3 University of Rwanda, Centre Hospitalier Universitaire de Kigali, Kigali, Rwanda

4 Muhimbili University of Health and Allied Sciences, Dar es Salaam, Tanzania
5 Department of Pediatric Oncology, Centre Hospitalier Universitaire de Kigali, Kigali, Rwanda

6 Yale University, New Haven, USA

7 Department of Pediatric Surgery, Muhimbili National Hospital, Dar es Salaam, Tanzania

8 Department of Pediatric Oncology, Muhimbili National Hospital, Dar es Salaam, Tanzania

9 Department of Paediatric Oncology, Oxford University Hospitals, Oxford, UK 


\section{Introduction}

\section{Background}

Nephroblastoma [Wilms' tumour (WT)] is the most common paediatric renal tumour, thought to arise from persistent metanephric cells or nephroblastomatosis [1]. Outcomes have improved significantly in high-income countries (HIC) over the past few decades with the 5-year survival rate increasing from $20 \%$ in the 1960 s to over $90 \%$ in the current decade [2-5]. This improvement is due to a multidisciplinary approach to treatment, and to a series of clinical trials led by two large study groups: the North American Children's Oncology Group via The National Wilms Tumour Study (NWTS) group, and the European Société Internationale d'Oncologie Pédiatrique (SIOP) group.

Outcomes are much poorer in low- and middle-income countries (LMIC), with survival in sub-Saharan Africa ranging from $11 \%$ in Sudan to $46 \%$ in Malawi [6]. The disparity in survival is multi-factorial: access to and availability of treatment; late presentation; limited resources and a paucity of trained staff; malnutrition; limited supportive care; abandonment of treatment; poverty; and perception of incurability [7-10]. Furthermore, the burden of childhood cancer predominates in LMIC (84\%), where $90 \%$ of the world's children live [11].

Oxford Paediatrics Linking Oncology Research with Electives (OxPLORE) is a collaboration founded in 2017 that unites HIC-based medical students with peers and junior doctors in LMIC settings during their elective placements. This novel research methodology of harnessing medical student power in medical research has been described elsewhere [12]. OxPLORE was conceived and developed at Oxford University, UK, by a group of medical students interested in global surgery and a trainee in paediatric surgery $(\mathrm{KF})$, with the support of the senior author (KL).

\section{Objectives}

The overall aim of OxPLORE is to foster bidirectional partnerships, enabling robust data collection to guide advancements in paediatric surgical and oncology care globally. In this paper, we report the centres' experiences of WT.

\section{Materials and methods}

Oxford medical students were recruited through advertising events preceding their elective placements. Students were provided with a letter of endorsement from the senior author (KL) to aid obtaining ethical approval; a data collection proforma; and were advised to engage with a local medical student or junior doctor. Electronic messaging and conferences enabled ongoing communication with collaborators after students returned from their electives. Data exchange occurred through security password-encoded excel spreadsheets. Deadlines were set for collation of data by Oxford and were analysed centrally (KF) given unpredictable working demands for collaborators in Tanzania and Rwanda.

Reporting of this study has been verified as per the STROBE (Strengthening the Reporting of Observational Studies in Epidemiology) checklist [13].

\section{Study design and setting}

The Phase One OxPLORE study is a cross-sectional observational study. Three collaborating centres were included in the study:

(1) Muhumbili National Hospital (MNH), Dar es Salaam, Tanzania

(2) Centre Hospitalier Universitaire de Kigali (CHUK), Kigali, Rwanda

(3) Oxford University Hospital (OUH), UK.

A retrospective medical record review was performed by collaborating centres of all children $(<16$ years $)$ presenting with WT between January 2016 and December 2017.

OUH uses an electronic patient registration system, allowing identification of children diagnosed with WT. There was no electronic system in MHN or CHUK, and patients were identified via inpatient ward registries, clinician notes and theatre logbooks.

\section{Participants}

There were no patient exclusion criteria other than age. Criteria for inclusion as a study site were close collaboration and transfer of research knowledge and skills between $\mathrm{H}$ - and LMIC medical students/junior doctors, senior clinician support from collaborating centre and local ethical approval.

\section{Variables and outcomes}

The primary outcome was survival to the point of discharge or death, whichever came first. Secondary outcomes included: patient demographics; stage of tumour at presentation; management (surgical and adjuvant medical); and post-operative morbidity. Post-operative morbidity was categorised as per the Clavien-Dindo classification: stage 1 (deviation from normal post-operative course 
without need for intervention); stage 2 (requiring pharmacological treatment); stage 3 (requiring surgical, radiological or endoscopic intervention); stage 4 (lifethreatening events needing intensive care or involving organ failure) [14].

\section{Data collection, management and analysis}

Data were retrospectively taken from patient files using a paper version of a standardised data collection tool. The tool was agreed by all three participating sites, allowing reliable comparison, before commencing data collection. Anonymised data were recorded electronically at each centre and analysed centrally using Graphpad Prism ${ }^{\circledR}$ version 8 (KF). Data are presented as median (range) unless otherwise stated and $p$ values of $<0.05$ considered statistically significant. One-way ANOVA was used to compare continuous variables, and Chi-squared tests to compare categorical variables. Missing data are acknowledged, but no statistical methods employed to account for them.

\section{Ethics/study oversight}

Regarding individual patients, there was no perceived risk or incentives, and consent was not required. Confidentiality was ensured and all data anonymised. Institutional endorsement was sought at each participating centre's review board, where the research protocol was reviewed and approved by the ethics board of CHUK (Ref: EC/ CHUK/680/2018), MNH (Ref: MNH/TRC/III/2018/018) and OUH (Ref: 4741).

\section{Results}

\section{Collaboration development}

The educational value of this collaboration for the junior researchers is evidenced through the presentation of results by medical students from the UK (SG) and Rwanda (EM), and surgical trainee $(\mathrm{KF})$ at international conferences in 2019. Moreover, students benefited from early experience in the challenges of international research, namely varying local protocols for ethical approval, time differences for communication, and limited time spent abroad. The costs of running phase one of the OxPLORE collaboration itself were by design minimal, and subsequent funding for attending presentations was sourced and applied for by students, which served as further experience in medical research. The collaboration also served as an experience of communication, on a peer-peer basis, junior-senior basis and low-high resource basis. The benefits to all parties on an educational and personal level were anecdotally commented on by all positively.

\section{Outcomes}

Further details and background of participating centres are shown in Table 1. There were 104 patients with WT treated in the three centres over the 2-year study period, with a larger patient volume in MNH ( $n=71$ cf. $n=26$ in CHUK and $n=7$ in OUH). Table 2 describes the core outcome set. Survival over the 2-year study period was $87 \%$ in MNH, $92 \%$ in CHUK and $100 \%$ in OUH at time of data closing (December 2018).

\section{Presentation}

More females with WT presented to the OUH cohort $(86 \%$ cf. $41 \%$ and $42 \%$ in $\mathrm{MNH}$ and CHUK, respectively). Age at presentation is shown in Fig. 1. There was no significant difference overall in age at presentation (one-way ANOVA, $F=0.2997, p=0.74$ ); however, the violin plots demonstrate a trend of younger age of presentation in the HIC setting [majority cluster of patients around the median in all three sites (40 months in MNH, 37 months in CHUK and 24 months in $\mathrm{OUH})]$.

Stage of disease at presentation was varied across the centres (Fig. 2). Stage in CHUK and OUH was widespread, whereas disease was more advanced in MNH. This was a significant finding when the data were categorised into stages I-II and compared with stages III-V ( $X^{2} 7.57$, $p=0.02$ ).

The presenting haemoglobin was similar in patients in the different centres, and no significant difference was found on one-way ANOVA $(F=1.144, p=0.32)$. Figure 3 shows the data distribution, where the range is noted to be more varied in the MNH cohort [median $9.88 \mathrm{~g} / \mathrm{dl}$ (range 5.4-15.3 g/dl)], compared with CHUK [median $10.1 \mathrm{~g} / \mathrm{dl}$ (range 6-10.4 g/dl)] and OUH [median $9.2 \mathrm{~g} / \mathrm{dl}$ (range $7.5-9.5 \mathrm{~g} / \mathrm{dl})]$.

\section{Treatment}

All patients had pre-operative chemotherapy (SIOP regimen). Most patients had nephrectomy (80\% MNH, $81 \%$ CHUK and $86 \%$ in OUH). Those who did not were deemed inoperable.

Post-operative morbidity (Clavien-Dindo scale) varied widely between the centres (Fig. 4), with more morbidity in the lower resourced settings when categories I-II were compared with III-IV $\left(X^{2} 33.72, p<0.0001\right)$. 


\section{Discussion}

The aim of this OxPLORE study was twofold: firstly, to establish and trial a medical student-facilitated research collaboration, using medical electives and existing links between the global north and south; and secondly to describe phase one results following the collaboration of three centres interested in comparing their experience of WT.

Student-led research collaborations have been shown to enable students to engage in high-quality studies with great impact, while gaining fundamental skills in study design and delivery [15]. Further, the bidirectional advantages of HIC-LMIC research partnerships are well described [16]. Worldwide, the highest burden of disease is in LMIC; however, medical research originating from these countries is limited. Collaboration between the different resourced settings can help overcome the challenges LMIC researchers face, including: few qualified researchers, less funding, limited infrastructure and lack of protected research time $[17,18]$. The utilisation of medical students to undertake research bypasses the issue of limited research time for overburdened doctors.

Validation of the success of this collaboration has not formally been conducted. Manifestation of valuable learning to individuals is demonstrated through formal presentations by collaborating medical students and contributions to this paper. This confers a 'result' impact of training, as per the Kirkpatrick model of evaluation [19]. Moreover, the personal relationships that have evolved from the first phase of the OxPLORE collaboration will continue to be nourished in the long-term and will provide the foundation for career-long collaboration between students in training and research. This contrasts with large global research collaborations, which can be faceless and do little to support or develop low- and middle-income partners.

OxPLORE has strived to address the power imbalance which can manifest in relationships between LMIC and HIC researchers by developing its research agenda collaboratively. Further, the collaboration with $\mathrm{MNH}$ was born from a longstanding partnership with $\mathrm{OUH}$, the

Table 1 Summary of participating centres

\begin{tabular}{|c|c|c|c|}
\hline & $\mathrm{MNH}^{\mathrm{a}}$ & $\mathrm{CHUK}^{\mathrm{b}}$ & $\mathrm{OUH}^{\mathrm{c}}$ \\
\hline Country population $^{\mathrm{d}}$ & $53,470,000$ & $11,610,000$ & $64,716,000$ \\
\hline \multirow[t]{2}{*}{ Under 5 mortality rate ${ }^{\mathrm{d}}$} & 54 & 37.9 & 4.3 \\
\hline & Per 1000 live births & Per 1000 live births & Per 1000 live births \\
\hline \multirow[t]{2}{*}{ Human development index ${ }^{\mathrm{e}}$} & 0.538 & 0.524 & 0.922 \\
\hline & 154/189 Countries & 158/189 Countries & 14/189 Countries \\
\hline \multirow[t]{2}{*}{ Referral centre for paediatric cancer } & Yes & Yes & Yes \\
\hline & National & National & Regional \\
\hline New paediatric cancer patients (year) & $400-500$ & $50-100$ & 107 \\
\hline Paediatric cancer treatment since & 2011 & 2012 & $>20$ years \\
\hline Paediatric cancer inpatient beds & 80 & 12 & 9 \\
\hline Number of paediatric cancer surgeons & 1 & 1 & 1 \\
\hline Number of paediatric oncologists & 1 & 1 & 4 \\
\hline Number of paediatric anaesthetists & 0 & 1 & 6 \\
\hline Paediatric intensive care & Yes & Yes & Yes \\
\hline Paediatric dieticians & No & Yes & Yes \\
\hline Nurse/patient ratio & $1: 6-10$ & $1: 8$ & $1: 1-3$ \\
\hline Treatment protocol & Modified SIOP & SIOP & SIOP \\
\hline Radiotherapy & Yes & No & Yes \\
\hline Pathology on site & Yes & Yes & Yes \\
\hline Free health care provision & Yes & No & Yes \\
\hline
\end{tabular}

${ }^{a}$ MNH, Muhumbili National Hospital, Dar es Salaam, Tanzania

${ }^{\mathrm{b}} \mathrm{CHUK}$, Centre Hospitalier Universitaire Kigali, Kigali, Rwanda

${ }^{\mathrm{O}} \mathrm{OUH}$, Oxford University Hospital, Oxford, UK

${ }^{\mathrm{d}}$ UNICEF 2018

${ }^{\mathrm{e}}$ United Nations Development Program 2017 
Table 2 Patients with WT presenting to the three collaborating centres over the 2-year study period

\begin{tabular}{|c|c|c|c|c|}
\hline & $\mathrm{MNH}^{\mathrm{a}}$ & $\mathrm{CHUK}^{\mathrm{b}}$ & $\mathrm{OUH}^{\mathrm{c}}$ & \\
\hline Number of patients & $N=71$ & $N=26$ & $N=7$ & - \\
\hline Female gender $(\%)$ & $29(41 \%)$ & $11(42 \%)$ & $6(86 \%)$ & $p=0.07 *$ \\
\hline Median (range) age on admission (months) & $40(4-113)$ & $37(6-104)$ & $24(14-145)$ & $p=0.74 * *$ \\
\hline \multicolumn{5}{|l|}{ Stage } \\
\hline $\mathrm{I}-\mathrm{II}$ & 10 & 10 & 3 & \multirow{2}{*}{$p=0.02 *$} \\
\hline III-V & 55 & 15 & 4 & \\
\hline Median (range) pre-operative haemoglobin ( $\mathrm{g} / \mathrm{dl}$ ) & $9.88(5.4-15.3)$ & $10.1(6-10.4)$ & $9.2(7.5-9.5)$ & $p=0.32 * *$ \\
\hline Pre-operative chemotherapy & $71(100 \%)$ & $26(100 \%)$ & $7(100 \%)$ & - \\
\hline Surgery & $57(80 \%)$ & $21(81 \%)$ & $6(86 \%)$ & $p=0.94 *$ \\
\hline \multicolumn{5}{|l|}{ Post-operative morbidity (Clavien-Dindo) } \\
\hline $\mathrm{I}-\mathrm{II}$ & 9 & 15 & 6 & \multirow[t]{2}{*}{$p<0.0001^{*}$} \\
\hline III-V & 48 & 6 & 0 & \\
\hline Survival & $62(87 \%)$ & $24(92 \%)$ & $7(100 \%)$ & $p<0.001^{*}$ \\
\hline
\end{tabular}

${ }^{a}$ Muhimbili National Hospital (MNH), Dar es Salaam, Tanzania

${ }^{\mathrm{b}}$ Centre Hospitalier Universitaire Kigali (CHUK), Rwanda

${ }^{\mathrm{c}}$ Oxford University Hospital (OUH), UK

*Chi-squared

**One-way ANOVA

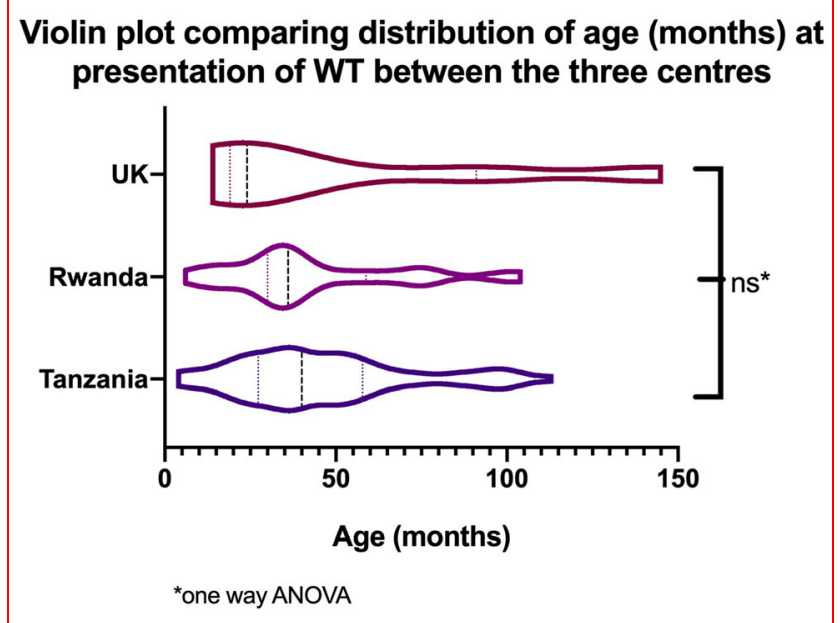

Fig. 1 Violin plot comparing distribution of age (months) at presentation of WT between the three centres

Oxford Tanzania Link [20], which shares experiences, supports medical learning and encourages adoption of the best medical practice.

\section{Survival}

Survival was higher in the HIC than the two LIC centres in the OxPLORE study. In this study, inadequate treatment does not seem to be a key contributor to disparities in mortality rate as comparative treatment options were available in all three centres. The lower chance of cure in less developed countries has been attributed to access to care, late diagnosis, inadequate resources for treatment, poor organisation and high rates of abandonment [21, 22]. Further, political instability also has negative effects on the reliable delivery of sustainable health programs in the African continent [23]. WT is a difficult cancer to cure in LMIC due to the multidisciplinary nature of the treatment involving surgery, imaging, pathology and oncology services. Although $\mathrm{MNH}$ and $\mathrm{CHUK}$ both have multidisciplinary paediatric oncology teams, this study could provide the evidence to initiate audit and analysis of the team efficacy and aid the departments in the advocacy of their needs.

Interestingly, OxPLORE reported survival rates in $\mathrm{MNH}$ $(87 \%)$ and CHUK $(92 \%)$ were higher than other studies in sub-Saharan Africa. Survival rates of $41 \%$ and $46 \%$ were reported in a similar retrospective study performed over a 2-year period at a single Kenyan centre [24] and Malawian centre, respectively [25]. The Malawian authors have further reported an associated haematological toxicity and treatment-related mortality in malnourished children following the initiation of SIOP pre-operative chemotherapy regimen [26]. Other outcomes in terms of 2-year event-free survival in North Africa [27] (73-87\%) and sub-Saharan Africa [7] (47\%) have been attributed to the studies analysing patients with low- or intermediate-risk tumours, 


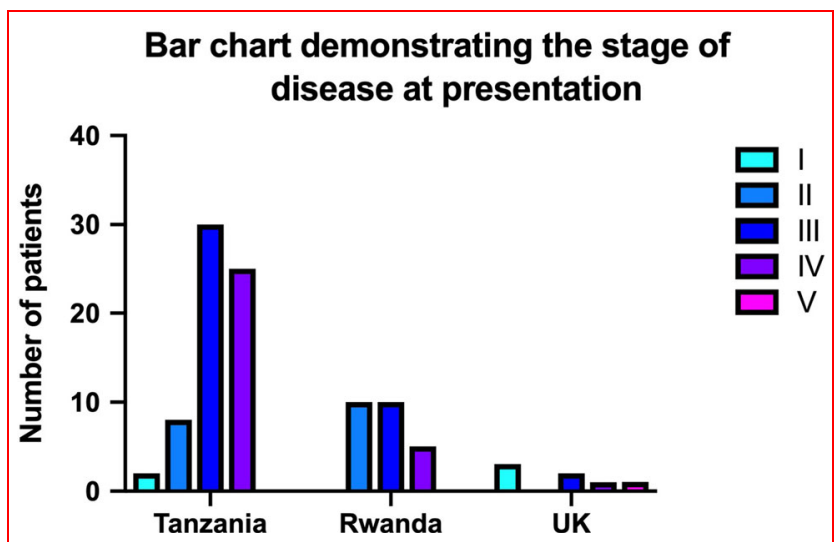

Fig. 2 Bar chart demonstrating the stage of disease at presentation

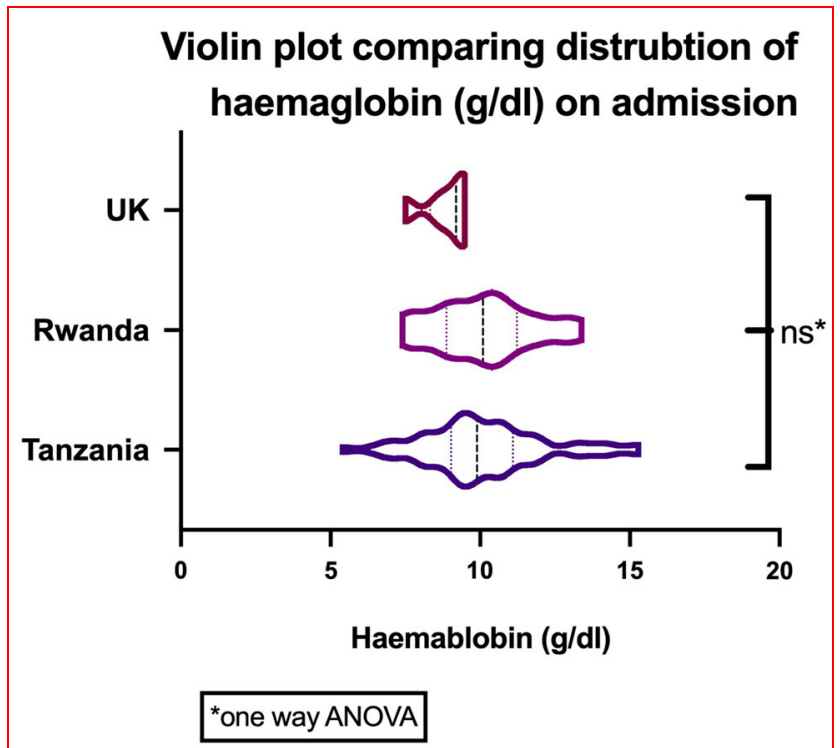

Fig. 3 Violin plot comparing distribution of haemoglobin $(\mathrm{g} / \mathrm{dl})$ on admission

without metastatic disease or progression post-operatively. Survival rates reported at 2-3 years follow-up from a population-based registry study in Eastern Africa were $38.7 \%$ in Harare, Zimbabwe; 15.8\% in Kampala, Uganda; and $22.9 \%$ in Nairobi, Kenya [28]. Our higher survival rate could be accounted for by the short-term follow-up of the OxPLORE study, and our definition of 'survival', as this will inevitably include patients who are palliative at the time of analysis. Unpublished survival data from $\mathrm{MNH}$ over a 2-year period report an 'alive and well' group of $47 \%$ at 12 months and $45 \%$ at 18 months.

Although not observed in this dataset, a key barrier to effective cancer treatment in children in sub-Saharan Africa is the abandonment of treatment. Regarding WT specifically, the period after surgical removal is the highest risk period [29], with misunderstanding of treatment plans and financial difficulty being identified as key causes for treatment abandonment in a Kenyan survey [30]. Loss to follow-up has been reported at $50 \%$ in Kenya, and countrywide efforts have been employed to enhance compliance. Introduction of a National Hospital Insurance Fund (NHIF) enrolment has been effective at increasing the numbers of patients completing successive phases of therapy and decreasing the risk of death [31].

\section{Presentation and treatment}

We reported differences in presentation between the three collaborating centres, including larger patient volume in MNH compared with CHUK and OUH. Patients tended to be younger in OUH which likely reflects their earlier presentation and the more advanced disease observed in the sub-Saharan African centres.

A further key difference between centres was post-operative morbidity. $\mathrm{MNH}$ had a much higher burden of severe post-operative complications (84\% with a ClavienDindo score III-V). Varying levels of intra- and post-operative facilities, resources and expertise to manage complications might account for this discrepancy. Furthermore, complex tumours of more advanced stage confer riskier and more challenging operations. Pre-operative morbidity status also influences subsequent outcome. Indeed, malnutrition at diagnosis results in increased treatment-related morbidity and treatment abandonment rate, and inferior event-free survival [32].

\section{Limitations of study}

There are several limitations in this study. Regarding study design and methodology, it was decided that a limited number of outcome measures would be recorded. This was due to predicted limitations of information in patient records and the fact that OxPLORE's inaugural study was also intended as a proof of concept of this multinational collaboration, so a simple study design is required. Furthermore, there were only a small number of patients in the OUH cohort, which limited the power of statistical analysis. The referral basin for OUH is a smaller (regional) population, compared to $\mathrm{MNH}$ and $\mathrm{CHUK}$ which serve as national referral centres for paediatric oncology in Tanzania and Rwanda, respectively. Finally, long-term follow-up of identified patients was not possible in this retrospective study which limits the interpretation of survival rates and ability of conclusions to be drawn regarding treatment abandonment and causation of morbidity and mortality.

Challenges faced, particularly in the LMIC setting, included missing data, access to data and obtaining ethical approval. As phase one methodology was retrospective, 
Fig. 4 Comparison of ClavienDindo classification
Comparion of Clavien-Dindo classification

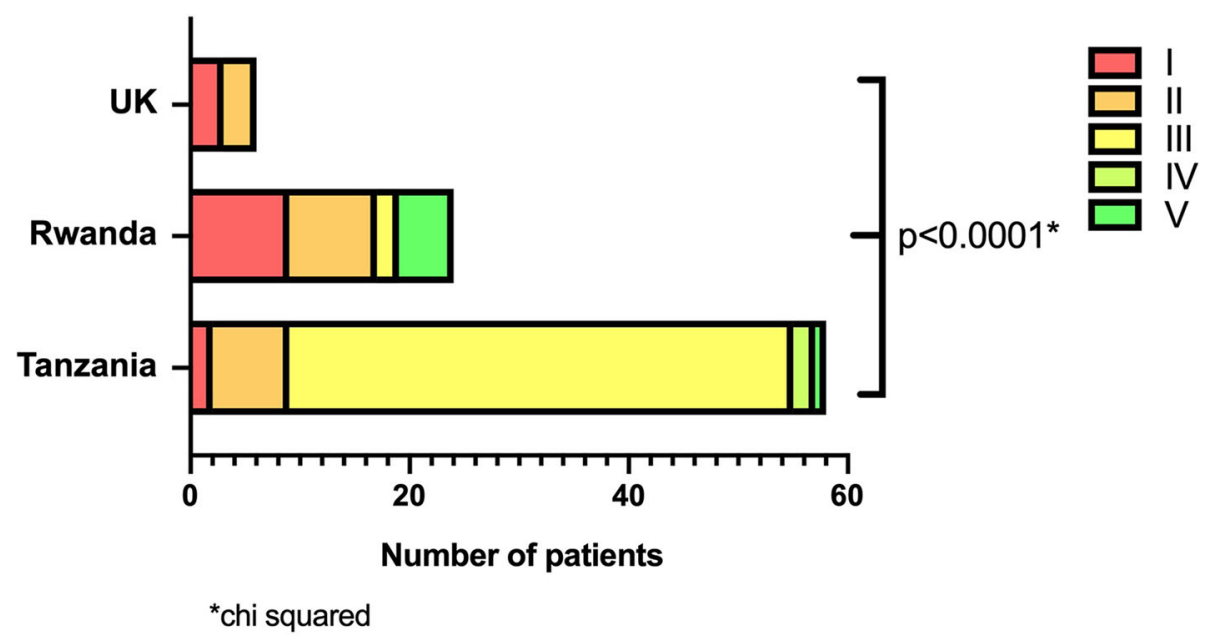

Fig. 5 Graphic demonstrating the improvements to childhood cancer pathway in a low resource setting
Graphic demonstrating the improvements to childhood cancer pathway in a low resource setting
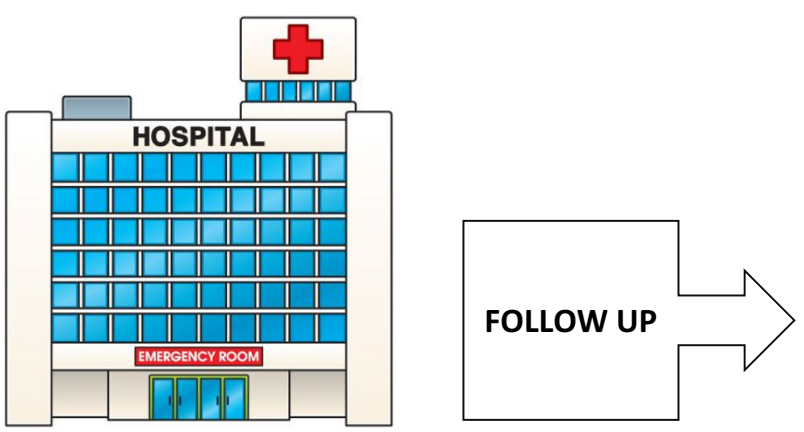

- Financial support

- Patient registries

- MDT approach

- Treatment protocol

- Focus on nutrition data quality is difficult to assess, so the generalisability and reliability of results may be affected.

\section{Recommendations}

Childhood cancer is a public health problem in developing countries. The majority of African children with cancer die without access to healthcare resources [23]. These same childhood cancers have excellent curative potential given the ideal treatment programmes, thus presenting an opportunity for improvement [33]. We recommend interventions at all stages of the child cancer journey, with focus on: early access to diagnosis; treatment in a multidisciplinary setting (involving surgical expertise, histology services, specialised nursing, pharmacy, laboratories, radiology, palliative care, infectious diseases, critical care and nutrition) via a standardised protocol and measures to address abandonment of treatment and loss to follow-up (Fig. 5).

\section{Conclusion}

Phase one of OxPLORE has proven the feasibility of medical student partnerships between HIC and LMIC to undertake collaboration, data collection and research outcome dissemination. This global collaboration and comparison describes the disparity in global outcomes of WT 
and advocates for the public health dilemma to be addressed as a global health priority.

Acknowledgements We thank all members of staff in MNH, CHUK and JRH and contributors to OxPLORE who have supported this project.

Open Access This article is distributed under the terms of the Creative Commons Attribution 4.0 International License (http://crea tivecommons.org/licenses/by/4.0/), which permits unrestricted use, distribution, and reproduction in any medium, provided you give appropriate credit to the original author(s) and the source, provide a link to the Creative Commons license, and indicate if changes were made.

\section{References}

1. Beckwith JB, Kiviat NB, Bonadio JF (1990) Nephrogenic rests, nephroblastomatosis, and the pathogenesis of Wilms' tumor. Pediatr Pathol 10:1-36

2. Metzger ML, Dome JS (2005) Current therapy for Wilms' tumor. Oncologist 10:815-826

3. D'Angio GJ, Breslow N, Beckwith JB et al (1989) Treatment of Wilms' tumor. Results of the Third National Wilms' Tumor Study. Cancer 64:349-360

4. Tournade MF, Com-Nougue C, de Kraker J et al (2001) Optimal duration of preoperative therapy in unilateral and nonmetastatic Wilms' tumor in children older than 6 months: results of the Ninth International Society of Pediatric Oncology Wilms' Tumor Trial and Study. J Clin Oncol 19:488-500

5. Rabeh W, Akel S, Eid T et al (2016) Wilms tumor: successes and challenges in management outside of cooperative clinical trials. Hematol Oncol Stem Cell Ther 9:20-25

6. Paintsil V, David H, Kambugu J et al (2015) The Collaborative Wilms Tumour Africa Project: baseline evaluation of Wilms tumour treatment and outcome in eight institutes in sub-Saharan Africa. Eur J Cancer 1:84-91

7. Israels $\mathrm{T}$ (2012) Wilms tumor in Africa: challenges to cure. Pediatr Blood Cancer 58:3-4

8. Israels T, Borgstein E, Jamali M et al (2009) Acute malnutrition is common in Malawian patients with a Wilms tumour: a role for peanut butter. Pediatr Blood Cancer 53(7):1221-1226

9. Mostert S, Arora RS, Arreola M et al (2011) Abandonment of treatment for childhood cancer: position statement of a SIOP PODC working group. Lancet Oncol 12(8):719-720

10. Gupta S, Yeh S, Martiniuk A et al (2013) The magnitude and predictors of abandonment of therapy in paediatric leukaemia in middle-income countries: a systematic review and metaanalysis. Eur J Cancer 49(11):2555-2564

11. Magrath I, Steliarova-Foucher E, Epelman S et al (2013) Improving cancer care for children and young people 2. Paediatric cancer in low-income and middle-income countries. Lancet Oncol 14(3):E104-E116

12. Mansfield RC, Gardner LCS, Ndukwe GOA et al (2013) Harnessing medical student power in global surgery research. Pediatr Surg Int 34(6):697-698

13. Vandenbroucke JP, von Elm E, Altman DG et al (2007) Strengthening the reporting of observational studies in epidemiology (STROBE): explanation and elaboration. PLoS Med 4(10):e297

14. Dindo D, Demartines N, Clavien PA (2004) Classification of surgical complications. A new proposal with evaluation in a cohort of 6336 patients and results of a survey. Ann Surg 240(2):205-213

15. Ahmed W, Mills E, Khaw R et al (2019) Collaborative student research efforts provide a solution to research wastage. BMJ 364:1795

16. Loukanova S, Prytherch H, Blank A et al (2014) Nesting doctoral students in collaborative North-South partnerships for health systems research. Glob Health Action 7:24070

17. Langer A, Diaz-Olavarrieta C, Berdichevsky K et al (2004) Why is research from developing countries underrepresented in international health literature, and what can be done about it? Bull World Health Organ 82:802-803

18. Trostle J, Simon J (1992) Building applied health research capacity in less-developed countries: problems encountered by the ADDR project. Soc Sci Med 35:1379-1387

19. Kirkpatrick D (1996) Great ideas revisited. Techniques for evaluating training programs. Train Dev 50:54-60

20. https://www.ouh.nhs.uk/get-involved/ok-links/default.aspx. Last accessed date 10 April 2019

21. Howard SC, Metzger ML, Wilimas JA et al (2008) Childhood cancer epidemiology in low-income countries. Cancer 112:461-472

22. Ribeiro RC, Steliarova-Foucher E, Magrath I et al (2008) Baseline status of paediatric oncology care in ten low-income or midincome countries receiving My Child Matters support: a descriptive study. Lancet Oncol 9:721-729

23. Harif M, Traore F, Hessissen L et al (2013) Challenges for paediatric oncology in Africa. Lancet Oncol 14(4):279-281

24. Njuguna F, Martijn HA, Kuremu RT et al (2017) Wilms tumour treatment options: perspectives from a low-income setting. J Glob Oncol 3(5):555-562

25. Israels T, Borgstein E, Pidini D et al (2012) Management of children with a Wilms tumour in Malawi, sub-Saharan Africa. J Pediatr Hematol Oncol 34:606-610

26. Israels T, Chagaluka G, Pidini D et al (2012) The efficacy and toxicity of SIOP preoperative chemotherapy in Malawian children with a Wilms tumour. Pediatr Blood Cancer 59:636-641

27. Moreira C, Nachef MN, Ziamati S et al (2012) Treatment of nephroblastoma in Africa: results of the first French African pediatric oncology group (GFAOP) study. Pediatr Blood Cancer 58:37-42

28. Joko-Fru WY, Parkin DM, Borok M et al (2018) Survival from childhood cancers in Eastern Africa: a population-based registry study. Int J Cancer 143:2409-2415

29. Friedrich P, Lam CG, Kaur G et al (2016) Determinants of treatment abandonment in childhood cancer: results from a global survey. PLOIS One 11(10):e0163090

30. Libes J, Oruko O, Abdallah F et al (2015) Risk factors for abandonment of Wilms tumor therapy in Kenya. Pediatr Blood Cancer 62(2):252-256

31. Axt J, Abdallah F, Axt M et al (2013) Wilms tumor survival in Kenya. J Pediatr Surg 48(6):1254-1262

32. Pribnow AK, Ortiz R, Baez L et al (2017) Effects of malnutrition on treatment-related morbidity and survival of children with cancer in Nicaragua. Pediatr Blood Cancer 64:e26590

33. El-Mallawany NK, Wasswa P, Mtete I et al (2017) Identifying opportunities to bridge disparity gaps in curing childhood cancer in Malawi: malignancies with excellent curative potential account for majority of diagnoses. Pediatr Hematol Oncol 34(5):261-274

Publisher's Note Springer Nature remains neutral with regard to jurisdictional claims in published maps and institutional affiliations. 